ИЗВЕСТИЯ АКАДЕМИИ НАУК ЭСТОНСКОИ ССР. ФИЗИКА * МАТЕМАТИКА PROCEEDINGS OF THE ACADEMY OF SCIENCES OF THE ESTONIAN SSR. PHYSICS * MATHEMATICS

$1986,35,3$

УДК 537.527

Э. TAMME, A. ШEPMAH

\title{
ЧИСЛЕННОЕ РЕЩЕНИЕ УРАВНЕНИЯ БОЛЬЦМАНА ДЛЯ ФУНКЦИИ РАСПРЕДЕЛЕНИЯ ЭЛЕКТРОНОВ ПО ЭНЕРГИЯМ В ГАЗОВОМ РАЗРЯДЕ
}

(Представил В. Хиюняков)

\section{1. Введение}

Одной из главных задач при численном моделировании разряда в газовой смеси является решение уравнения Больцмана для функции распределения электронов по энергиям [1 $]$. При большой величине напряженности электрического поля эта функция существенно отличается от больцмановской. Константы скорости реакций, происходящих в разряде с участием электронов, определяются сверткой соответствующих сечений с функцией распределения. В результате эти константы, как показывает расчет $\left[{ }^{2-4}\right]$, обладают сильной зависимостью от напряженности электрического поля и от концентрации возбужденных компонент плазмы. Среди этих констант выделим константы скорости важнейших реакций, приводящих к размножению и гибели электронов. Таким образом, правильность решения уравнения Больцмана является одной из главных предпосылок для создания надежной математической модели газового разряда.

В данной работе описывается алгоритм решения уравнения Больцмана, использовавшийся нами при создании кинетической модели газового разряда в смеси $\mathrm{HCl} / \mathrm{Xe} / \mathrm{He}\left[{ }^{4-6}\right]$. В уравнении Больцмана учитываются процессы упругих, неупругих и сверхупругих столкновений электронов с атомами Не, Хе и с молекулами $\mathrm{HCl}$, а также электрон-электронные столкновения. Полученные модельные результаты по временному поведению возбужденных компонент плазмы разряда для смесей различного состава $\left[{ }^{5,6}\right]$ находятся в удовлетворительном согласии с экспериментальными по абсорбционному зондированию [ㄱ]. Это дает основание считать полученные с помощью предлагаемого алгоритма результаты достаточно надежными для рассматриваемого класса задач.

\section{2. Уравнение Больцмана}

Для интересующих нас газовых разрядов в смесях с давлением в несколько атмосфер время релаксации функции распределения электронов по энергиям к квазистационарному виду (меньше или порядка 100 пс $\left.\left[{ }^{8}\right]\right)$ значительно меньше типичных времен химической кинетики ( 1-10 нс в нашем случае). Это дает возможность использовать квазистационарное уравнение Больцмана $[1,9]$ для релаксированной функции распределения $f(u)$

$$
\frac{d}{d u}\left[A(u) \frac{d}{d u} f(u)+B(u) f(u)\right]+S(u)=0,
$$

где $u$-энергия электронов, эВ, 


$$
\begin{aligned}
A(u)= & \frac{1}{3} E^{2} u Q_{1 m}^{-1}(u)+\frac{2 m k T}{e} u^{2} Q_{2 m}(u)+2 \Gamma_{e e}[e] g(u), \\
B(u)= & 2 m Q_{2 m}(u) u^{2}+3 \Gamma_{e e}[e] h(u), \\
S(u)= & \sum_{j=1}^{7}\left\{c_{j}\left[\left(u+u_{j}\right) f\left(u+u_{j}\right) Q_{j}\left(u+u_{j}\right)-u f(u) Q_{j}(u)\right]-\right. \\
& \left.-c_{j}^{\prime}\left[u f\left(u-u_{j}\right) Q_{j}(u)-\left(u+u_{j}\right) f(u) Q_{j}\left(u+u_{j}\right)\right]\right\} .
\end{aligned}
$$

Функция распределения удовлетворяет условию нормировки

$$
\int_{0}^{\infty} u^{1 / 2} f(u) d u=1
$$

В (2) $E$ - напряженность электрического поля, $\quad c_{2}^{\prime}=c_{4}^{\prime}=c_{5}^{\prime}=c_{6}^{\prime}=0$, $[e]-$ концентрация электронов, $c_{1}=c_{2}=[\mathrm{Xe}], c_{3}=c_{4}=[\mathrm{He}]-$ концентрации атомов Хе и Не в основных состояниях, $c_{5}=c_{1}^{\prime} \cdot g \mathrm{xe}=\left[\mathrm{Xe}^{*}\right]$, $c_{6}=c_{3}^{\prime} \cdot g^{\mathrm{He}}=\left[\mathrm{He}^{*}\right] \quad$ концентрации Хе и Не в нижних метастабильных состояниях (соответственно ${ }^{3} P_{2},{ }^{3} P_{0}$ и $\left.{ }^{3} S_{1},{ }^{1} S_{0}\right), g_{\text {хе }}=6$ и $g_{\mathrm{He}}=4-$ кратности вырождения этих состояний, $c_{7}=[\mathrm{HCl}], c^{\prime}{ }_{7}=[\mathrm{HCl}(v)]-$ концентрации молекул $\mathrm{HCl}$ в основном и первом колебательно-возбужденном состояниях, $Q_{j}(u)$ и $u_{j}$ - сечения и пороговые энергии соответствующих реакций: $j=1$ - возбуждение Хе в метастабильные состояния, $j=2$ - ионизация Хе из основного состояния, $j=3$ и $4-$ аналогичные процессы для Не, $j=5$ и 6 - ионизация $\mathrm{Xe}^{*}$ и $\mathrm{He}^{*}$ соответственно, $j=7$ - колебательное возбуждение $\mathrm{HCl}$.

$$
\begin{gathered}
Q_{1 m}(u)=[\mathrm{He}] Q_{m, \mathrm{He}}(u)+[\mathrm{Xe}] Q_{m, \mathrm{Xe}}(u), \\
Q_{2 m}(u)=[\mathrm{He}] Q_{m, \mathrm{He}}(u) / M_{\mathrm{He}}+[\mathrm{Xe}] Q_{m, \mathrm{Xe}}(u) / M_{\mathrm{Xe}}
\end{gathered}
$$

$Q_{m, \text { не}}, Q_{m, \text { хе }}-\quad$ сечения упругих столкновений электронов с соответствующими атомами, $M_{\mathrm{He}}$ и $M_{\text {хе }}$ - их массы. Влияние упругих столкновений электронов с молекулами $\mathrm{HCl}$ в интересующих нас смесях с малым содержанием этого компонента мало́ и поэтому не учитывается в (1), (2) (сечение для этого процесса можно найти в $\left[{ }^{10}\right]$; литературные источники по сечениям остальных реакций приведены в $\left.\left[{ }^{4}\right]\right)$. Кулоновские интегралы $g(u)$ и $h(u)$ определяются формулами $\left[{ }^{9}\right]$

$$
\begin{gathered}
g(u)=\int_{0}^{u} x^{3 / 2} f(x) d x+u^{3 / 2} \int_{u}^{\infty} f(x) d x, \quad h(u)=\int_{0}^{u} x^{1 / 2} f(x) d x, \\
\Gamma_{e e}=\frac{e^{2}}{24 \pi \varepsilon} \ln \frac{12 \pi\left(\frac{2}{3} \varepsilon \bar{u} e\right)^{3 / 2}}{e^{3}[e]^{1 / 2}},
\end{gathered}
$$

$\bar{u}=\int_{u}^{\infty} u^{3 / 2} f(u) d u-\quad$ средняя энергия электронов, $m$ и $e-$ их масса и заряд, $\varepsilon$ - диэлектрическая пронишаемость вакуума, $k-$ постоянная Больцмана, $T=300 \mathrm{~K}$ - температура газа. Благодаря слабой зависимости кулоновского логарифмического фактора $\Gamma_{e е}$ от концентрации электронов [e] и их средней энергии $\bar{u}$, в расчетах использовалось фиксированное значение $\Gamma_{e e}=3,16 \cdot 10^{-17}$ Дж ${ }^{2} \cdot \mathrm{M}^{2} / \mathrm{K}^{2}$, соответствующее типичным для рассматриваемых разрядов условиям: $[e] \approx 10^{15} \mathrm{~cm}^{-3}$ $\bar{u} \approx 3,5$ эВ.

Функция распределения электронов по энергиям, найденная из урав 
нения Больцмана (1), используется для вычисления конста̄нт скоростй реакций с участием электронов,

$$
k_{j}=\left(\frac{2 e}{m}\right)^{1 / 2} \int_{0}^{\infty} Q_{j}(u) u f(u) d u,
$$

входящих в кинетические уравнения, и других величин, характеризующих газовый разряд (см. $\left.\left[{ }^{4}\right]\right)$.

\section{3. Алгоритм решения уравнения Больцмана}

Для приближенного решения уравнения Больцмана (1), (2) и вычисления констант реакций (4) нами была составлена на языке ФОРТРАН подпрограмма BOLTZ. В качестве основы использовался алгоритм, описанный в [ $\left.{ }^{1}\right]$. Для контроля точности и выбора наилучшего способа решения были составлены несколько вариантов этой подпрограммы и проведены с ними численные экспернменты. Удовлетворительные результаты получены подпрограммой, написанной на основе следующего алгоритма.

Рассмотрим вначале уравнение Больцмана (1), (2) без слагаемых, описывающих электрон-электронные столкновения и содержащих кулоновские интегралы $g(u)$ и $h(u)$. Способом, описанным ниже, выбираем максимальную энергию, до которой производится счет, $u_{m} \leqslant 50$ эВ и решаем это уравнение на отрезке $0 \leqslant u \leqslant u_{m}$, причем считаем, что $f(u)=0$ при $u>u_{m}$. Уравнение (1) дискретизнруем стандартным способом. Выбираем узлы

$$
u_{0}=0, \quad u_{l}=u_{l-1}+\Delta u_{l}, \quad l=1,2, \ldots, 104,
$$

где $\Delta u_{l}=\Delta / 2$ при $l=1, \ldots, 8$ и $\Delta u_{l}=\Delta$ при $l=9, \ldots, 104 ; \Delta=u_{m} / 100$. Приближенные значения решения $f_{l}=f\left(u_{l}\right)$ находятся из системы линейных уравнений

$$
\begin{gathered}
\left(A_{l}-B_{l-1}\right) f_{l-1}-\left(A_{l+1}+A_{l}\right) f_{l}+\left(A_{l+1}+B_{l+1}\right) f_{l+1}+S_{l}=0, \\
\text { при } l=1, \ldots, 7,9, \ldots, 104, \\
\left(A_{l}-2 B_{l-1}\right) f_{l-1}-\left(2 A_{l+1}+A_{l}-1,5 B_{l}\right) f_{l}-\left(2 A_{l+1}+B_{l+1}\right) f_{l+1}+1,5 S_{l}=0, \\
\text { при } l=8,
\end{gathered}
$$

где $f_{105}=0, \quad A_{l}=A\left(u_{l}-\Delta u_{l} / 2\right) /\left(\Delta u_{l}\right)^{2}, \quad B_{0}=0, \quad B_{l}=0,5 B\left(u_{l}\right) / \Delta u_{l}, \quad S_{l}=$ $=S\left(u_{l}\right)$. В выражении $S_{l}=S\left(u_{l}\right)$ значения $f\left(u_{l}+u_{j}\right)$ и $f\left(u_{l}-u_{j}\right)$ аппроксимируются с помощью линейной интерполяции значений функции $f$ в двух узлах, между которыми находятся соответственно $u_{l}+u_{j}$ и $u_{l}-u_{j}$. Функции $Q_{1 m}(u), Q_{2 m}(u)$ и $Q_{j}(u), \quad j=1, \ldots, 7$, хранятся в памяти ЭВМ в виде массивов значений на некотором базисном множестве точек; выбор нужного значения осуществляется также с помощью линейной интерполяции.

Так как система (5) однородна, одно значение $f_{l}$ можно зафиксировать. Мы приняли $f_{104}=10^{-25}$ и решали систему (5) методом Гаусса, предварительно преобразовав ее матрнцу к верхнему треугольному виду. При этом учитывались нули в левом нижнем углу.

Длина отрезка $u_{m}$ выбиралась следующим образом. При первом обращении к BOLTZ принималось $u_{m}=50$ эВ. Если при решении системы (5) некоторое значение $f_{l}$ получается отрицательным или больше $10^{25}$,

* Функция $f(u)$ достаточно быстро стремится к нулю при $u \rightarrow \infty$, что позволяет использовать это приближение в интегралах типа (4) при условин $u_{m} \gg \bar{u}$. 
$u_{m}$ уменьшается в $2^{1 / 3}$ раз и система решается снова. Это повторяется до тех пор, пока все $f_{l} \leqslant 10^{25}$. При решении уравнения Больцмана совместно с системой кинетических уравнений, описывающих плазмо-химические процессы в разряде, и с уравнениями электрической цепи возникает необходимость в повторном обращении к BOLTZ с несколько измененными значениями напряженности поля $E$ и конщентраций компонент. В этом случае на первом шаге $u_{m}$ принимается равным последнему значению из предыдущего обращения и при необходимости уменьшается в последующих шагах описанным выше образом. Если же $f_{0} \leqslant 10^{7}$ и $u_{m}<50$ эВ , то $u_{m}$ увеличивается в $2^{1 / 3}$ раза. Таким образом, $u_{m} \leqslant 50$ эВ выбирается так. чтобы $10^{32}<f_{0} / f_{104} \leqslant 10^{50}$.

После решения системы (5), нспользуя формулу Симпсона, функция распрелеления $f(u)$ нормируется и вычисляются константы скорости реакций $k_{j}$.

Проверка показала, что получаемые таким путем значения констант скорости имеют относительную погрешность менее $5 \%$. Лишь в случае малых $k_{j}$ (менее $10^{-15} \mathrm{~cm}^{3} / \mathrm{c}$ ) погрешность несколько больше. Погрешность определялась сравнением с $k_{j}$, получаемыми при увеличении числа интервалов деления вдвое и при той же точке изменения шага $u_{m} / 25$.

В численных экспериментах шаг изменялся и в других местах. Кроме того, делались попытки изменить шаг дважды и трижды в интервале $\left(0, u_{m}\right)$, но сушественного уменьшения погрешности при приблизительно том-же числе уравнений и неизвестных достигнуто не было.

Рассмотрим теперь уравнение Больцмана (1), (2) с учетом электронэлектронных столкновений. Так как в этом случае уравнение нелинейно, то для его решения использовался следующий итерационный метод. На первом шаге электрон-электронные столкновения не учитывались и система (5) решалась описанным выше способом. Пользуясь полученным нормированным решением составлялись, используя для интегрирования формулу трапеции, таблицы функший $g(u)$ и $h(u)$. С их помощью вычислялись новые коэффициенты $A_{1}$ и $B$, система (5) решалась снова, определялась новая нормированная функция распределения, составлялись новые таблицы функций $g(u)$ и $h(u)$ и т. д. Для контроля сходимости итерационной процедуры использовались вычисляемые на каждом шаге константы $k_{\text {; }}$ с средняя энергия $\bar{u}$ и подвижность электронов. Қак показал расчет, такой итерационный метод при используемых нами начальных данных сходился довольно быстро.

\section{4. Численные результаты}

Приведем в виде таблиц некоторые численные результаты, полученные подпрограммой BOLTZ. Расчет проводился для смеси $\mathrm{HCl} / \mathrm{Xe} / \mathrm{He}=$ $=1 / 10 / 920$ при общем давлении 2,2 атм. Концентрация возбужденных компонент и электронов изменялась, причем піредполагались выполненными соотношения [Хe* $]=10\left[\mathrm{He}^{*}\right]=0,2[\mathrm{HCl}(v)]$. Для выяснения роли сверхупругих и электрон-электронных столкновений уравнение Больцмана (1) решалось в трех вариантах. В варианте $A$ эти процессы не учитывались (т. е. слагаемые, содержащие $c^{\prime}{ }_{j}$ и $\Gamma_{e e}$ в (1), (2) игнорировались). В варианте $B$ не учитывались электрон-электронные столкновения. В варианте $C$ принимались во внимание и сверхупругие, и электронэлектронные столкновения, причем прелполагалось, что $[e]=\left[\mathrm{Xe}^{*}\right]$. Для решения уравнения Больцмана (1) на ЭВМ ЕС-1060 требовалось в случае варианта $A$ менее 0,5 сек, в случае варианта $B$ около 2 сек и в случае варианта $C$ от 6 до 30 сек в зависимости от числа итераций.

Как видно из табл. 1 и 2, вклад сверхупругих и электрон-электронных столкновений весьма существен при напряженностях электрического 
Функция распределения электронов по энергиям $f(u)$ при $\left[\mathrm{Xe}^{*}\right]=10^{15} \mathrm{~cm}^{-3}$

\begin{tabular}{|c|c|c|c|c|c|c|c|c|}
\hline \multirow{2}{*}{$\begin{array}{c}\text { Напря- } \\
\text { жен- } \\
\text { ность } \\
\text { поля, } \\
\text { кВ/см }\end{array}$} & \multirow{2}{*}{$\begin{array}{c}\text { Вари- } \\
\text { ант }\end{array}$} & \multicolumn{7}{|c|}{$u, \vartheta \mathrm{B}$} \\
\hline & & 0,5 & 3 & 5 & 10 & 15 & 20 & 40 \\
\hline$E=4$ & $\begin{array}{l}A \\
B \\
C\end{array}$ & $\begin{array}{l}0,13 \\
0,13 \\
0,16\end{array}$ & $\begin{array}{l}0,086 \\
0,086 \\
0,085\end{array}$ & $\begin{array}{l}0,050 \\
0,050 \\
0,046\end{array}$ & $\begin{array}{l}0,073 \\
0,074 \\
0,072\end{array}$ & $\begin{array}{l}3,0 \cdot 10^{-4} \\
3,0 \cdot 10^{-4} \\
4,5 \cdot 10^{-4}\end{array}$ & $\begin{array}{l}3 \cdot 10^{-6} \\
4 \cdot 10^{-6} \\
9 \cdot 10^{-6}\end{array}$ & $\begin{array}{l}1 \cdot 10^{-20} \\
1 \cdot 10^{-13} \\
4 \cdot 10^{-13}\end{array}$ \\
\hline$E=2$ & $\begin{array}{l}A \\
B \\
C\end{array}$ & $\begin{array}{l}0,37 \\
0,34 \\
0,34\end{array}$ & $\begin{array}{l}0,10 \\
0,10 \\
0,09\end{array}$ & $\begin{array}{l}0,021 \\
0,022 \\
0,029\end{array}$ & $\begin{array}{l}1,4 \cdot 10^{-4} \\
1,7 \cdot 10^{-4} \\
9,3 \cdot 10^{-4}\end{array}$ & $\begin{array}{l}6 \cdot 10^{-8} \\
5 \cdot 10^{-7} \\
7 \cdot 10^{-6}\end{array}$ & $\begin{array}{l}2 \cdot 10^{-12} \\
5 \cdot 10^{-8} \\
6 \cdot 10^{-8}\end{array}$ & $\begin{array}{r}10^{-42} \\
3 \cdot 10^{-15} \\
3 \cdot 10^{-15}\end{array}$ \\
\hline$E=1$ & $\begin{array}{l}A \\
B \\
C\end{array}$ & $\begin{array}{l}0,98 \\
0,97 \\
0,60\end{array}$ & $\begin{array}{l}0,009 \\
0,015 \\
0,058\end{array}$ & $\begin{array}{l}3 \cdot 10^{-5} \\
8 \cdot 10^{-4} \\
9 \cdot 10^{-3}\end{array}$ & $\begin{array}{l}7 \cdot 10^{-13} \\
7 \cdot 10^{-5} \\
3 \cdot 10^{-5}\end{array}$ & $\begin{array}{l}2 \cdot 10^{-22} \\
5 \cdot 10^{-8} \\
1 \cdot 10^{-7}\end{array}$ & $\begin{array}{c}10^{-34} \\
8 \cdot 10^{-7} \\
7 \cdot 10^{-8}\end{array}$ & $\begin{array}{c}0 \\
2 \cdot 10^{-14} \\
3 \cdot 10^{-15}\end{array}$ \\
\hline
\end{tabular}

Таблица 2

Константы скорости реакций $\mathrm{Xe}+e \rightarrow \mathrm{Xe}^{*}+e\left(k_{1}\right), \mathrm{Xe}+e \rightarrow \mathrm{Xe}^{+}+2 e\left(k_{2}\right)$, $\mathrm{Xe}^{*}+e \rightarrow \mathrm{Xe}^{+}+2 e\left(k_{5}\right)$

\begin{tabular}{|c|c|c|c|c|c|c|c|c|}
\hline & \multirow{2}{*}{$\begin{array}{c}E, \\
\kappa \mathrm{B} / \mathrm{cm}\end{array}$} & \multirow{2}{*}[\mathrm{Xe}^{*}]{$=0$} & \multicolumn{3}{|c|}{$\left[\mathrm{Xe}^{*}\right]=10^{14} \mathrm{~cm}^{-3}$} & \multicolumn{3}{|c|}{$\left[\mathrm{Xe}^{*}\right]=10^{15} \mathrm{~cm}^{-3}$} \\
\hline & & & $A$ & $B$ & $C$ & $A$ & $B$ & $C$ \\
\hline $\begin{array}{l}k_{1}, \\
\mathrm{~cm}^{3} / \mathrm{c}\end{array}$ & $\begin{array}{l}4 \\
2 \\
1\end{array}$ & $\begin{array}{l}3,9 \cdot 10^{-10} \\
5,5 \cdot 10^{-12} \\
3 \cdot 10^{-19}\end{array}$ & $\begin{array}{l}3,9 \cdot 10^{-10} \\
5,4 \cdot 10^{-12} \\
3 \cdot 10^{-19}\end{array}$ & $\begin{array}{l}3,9 \cdot 10^{-10} \\
5,4 \cdot 10^{-12} \\
3 \cdot 10^{-14}\end{array}$ & $\begin{array}{l}3,9 \cdot 10^{-10} \\
8,3 \cdot 10^{-12} \\
3 \cdot 10^{-14}\end{array}$ & $\begin{array}{l}3,7 \cdot 10^{-10} \\
4,8 \cdot 10^{-12} \\
3 \cdot 10^{-19}\end{array}$ & $\begin{array}{l}3,7 \cdot 10^{-10} \\
5,9 \cdot 10^{-12} \\
2 \cdot 10^{-12}\end{array}$ & $\begin{array}{l}4,0 \cdot 10^{-10} \\
3,4 \cdot 10^{-11} \\
1 \cdot 10^{-12}\end{array}$ \\
\hline $\begin{array}{l}k_{2} \\
\mathrm{~cm}^{3} / \mathrm{c}\end{array}$ & $\begin{array}{l}4 \\
2 \\
1\end{array}$ & $\begin{array}{l}1,2 \cdot 10^{-10} \\
8 \cdot 10^{-14} \\
5 \cdot 10^{-26}\end{array}$ & $\begin{array}{l}1,2 \cdot 10^{-10} \\
8 \cdot 10^{-14} \\
5 \cdot 10^{-26}\end{array}$ & $\begin{array}{l}1,2 \cdot 10^{-10} \\
1,0 \cdot 10^{-13} \\
6 \cdot 10^{-15}\end{array}$ & $\begin{array}{l}1,3 \cdot 10^{-10} \\
1,9 \cdot 10^{-13} \\
6 \cdot 10^{-15}\end{array}$ & $\begin{array}{l}1,1 \cdot 10^{-10} \\
6 \cdot 10^{-14} \\
4 \cdot 10^{-26}\end{array}$ & $\begin{array}{l}1,2 \cdot 10^{-10} \\
3,3 \cdot 10^{-13} \\
5 \cdot 10^{-13}\end{array}$ & $\begin{array}{l}1,7 \cdot 10^{-10} \\
3,3 \cdot 10^{-12} \\
1 \cdot 10^{-13}\end{array}$ \\
\hline $\begin{array}{l}k_{5} \\
\mathrm{~cm}^{3} / \mathrm{c}\end{array}$ & $\begin{array}{l}4 \\
2 \\
1\end{array}$ & $\begin{array}{l}7,0 \cdot 10^{-8} \\
1,4 \cdot 10^{-8} \\
4 \cdot 10^{-11}\end{array}$ & $\begin{array}{l}7,1 \cdot 10^{-8} \\
1,4 \cdot 10^{-8} \\
4 \cdot 10^{-11}\end{array}$ & $\begin{array}{l}7,0 \cdot 10^{-8} \\
1,4 \cdot 10^{-8} \\
5 \cdot 10^{-11}\end{array}$ & $\begin{array}{l}6,9 \cdot 10^{-8} \\
1,5 \cdot 10^{-8} \\
2 \cdot 10^{-10}\end{array}$ & $\begin{array}{l}6,9 \cdot 10^{-8} \\
1,3 \cdot 10^{-8} \\
4 \cdot 10^{-11}\end{array}$ & $\begin{array}{l}6,9 \cdot 10^{-8} \\
1,4 \cdot 10^{-8} \\
1 \cdot 10^{-9}\end{array}$ & $\begin{array}{l}6,5 \cdot 10^{-8} \\
2,3 \cdot 10^{-8} \\
5 \cdot 10^{-9}\end{array}$ \\
\hline
\end{tabular}

поля $|E|<4$ кВ/см и при концентрациях возбужденных компонент и электронов, превосходящих $10^{14} \mathrm{~cm}^{-3}$. Такие условия реализуются на определенных стадиях разряда. По сравнению с вариантом $A$ константы скорости отмеченных и ряда других реакций в варианте $C$ при указанных условиях возрастают на несколько порядков. В проведенных нами расчетах разрядов в данной смеси такие изменения констант реакций приводят к существенному изменению временного хода концентраций компонент и к увеличению мгновенных значений некоторых из них в несколько раз **.

** Отметим, что при моделировании разряда в рассматриваемой газовой смеси приходится пренебрегать вкладом сверхупругих и электрон-электронных столкновений при напряженностях поля $|E|<1$ кВ/см. Слагаемые в (1), (2), описывающие электронэлектронные столкновения, получены в предположении малости передаваемого при столкновении нмпульса [11]. При малых $|E|$ и $\bar{u} \Gamma_{c e}$ в (3) становнтся отрицательным, что свидетельствует о нарушении этого условия. В этой же области изменения $E$ слагаемые, связанные со сверхупругими соударениями, приводят к неустойчивости алгоритма решения уравнения Больцмана. Заметнм, однако, что временные интервалы, в течение которых в реальном разряде $|E|<1$ кВ/см, коротки ( 1 нс) в представляющих интерес стадиях разряда. Это позволяет надеяться, что вносимые таким приближением искажения малы, 
1. Смит K., Томсон $P$. Численное моделирование газовых лазеров. М., «Мир», 1981.

2. Maeda. M., Takahashi, A., Mizunami, T., Miyazoe, Y. Jap. J. Appl. Phys., 21, № 8, $1161-1169(1982)$

3. Демьянов А. В., Кочетов Н. В., Напартович А. П., Старостин А. Н., Таран М. Д. Препринт ИАЭ-4093/12. М., 1985.

4. Мийдла П., Тамме Э., Шерман А. Изв. АН ЭССР. Физ. Матем., 35, № 1, 77-85 (1986).

5. Sherman, A., Miidla, P., Tamme, E. XVII Intern. Conf. Phenomena in Ionized Gases, Contributed Papers. Budapest (Hungary), 1985, 849-850.

6. Мийдла П. Х.. Пеэт В. Э., Соркина Р. А., Тамме Э. Э., Треияалов А. Б., Шерман A. B. Квантовая электроника (в печати).

7. Peet. V. E., Tsarenko, S. A., Sorkina, R. A., Treshchalov, A. B. 3rd Svmp. Optical Snectroscopy. Abstracts of Invited Lectures and Posters. Reinhardsbrunn (GDR), 1984, 32.

8. Johnson, T. H., Palumbo, L. J. Hunter II, A. M. J. Quant. Electron., QE-15, № 5, 289-301 (1979).

9. Shlie, L. A. J. Appl. Phys., 47. № 4, $1398-1407$ (1976).

10. Гальцев В. Е. Демьянов А. В., Кочетов И. В., Певгов В. Г., Шарков В. Ф. Препринт ИАЭ-3156. М., 1979.

11. Смирнов Б. М. Физика слабононизованного газа. М., «Наука», 1972.
Ннститут физики
Академии наук Эстонской ССР
Поступила в редакцию
16/XII 1985

\section{E. TAMME, A. SERMAN}

\section{BOLTZMANNI VORRANDI NUMBRILINE LAHENDAMINE ELEKTRONIDE JAOTUSFUNKTSIOONI LEIDMISEKS GAASLAHENDUSES}

On kirjeldatud kiiret algoritmi Boltzmanni võrrandi lahendamiseks elektronide jaotusfunktsiooni leidmisel gaaslahenduses. Elektronide elastsete ia mitteelastsete pōrgete kōrval gaasi aatomite ja molekulidega on Boltzmanni vōrrandis arvestatud ülielastsete ja elektron-elektron-põrgete panust. Gaasisegu $\mathrm{HCl} / \mathrm{Xe} / \mathrm{He}=1 / 10 / 920, n=2,2$ atm näite varal demonstreeritakse, et piirkonnas, kus elektrivälia tugevus $|E|<4 \mathrm{kV} / \mathrm{cm}$ ning ergastatud komponentide ja elektronide kontsentratsioon ïletab $10^{14} \mathrm{~cm}^{-3}$, etendavad kaks viimast protsessi olulist osa, põhjustades mōnede reaktsioonide kiiruse konstantide kasvu mitme suurusjärgu võrra.

\section{E. TAMME, A. SHERMAN}

\section{NUMERICAL SOLUTION OF THE BOLTZMANN EQUATION FOR ELECTRON DISTRIBUTION FUNCTION IN GAS DISCHARGE}

A fast algorithm of soluting the Boltzmann equation for the electron distribution function in gas discharge is described. Besides the processes of elastic and inelastic collisions of electrons with atoms and molecules, superelastic and electron-electron collisions are taken into account. The algorithm uses the Gauss' method of matrix equation solution. By making use as an example $\mathrm{HCl} / \mathrm{Xe} / \mathrm{He}$ gas mix $(1 / 10 / 920$, $p=2.2 \mathrm{~atm})$ it is shown that in the region of electrical intensities $|E|<4 \mathrm{kV} / \mathrm{cm}$ and concentrations of excited components and electrons exceeding $10^{14} \mathrm{~cm}^{-3}$, the last two processes play significant role, increasing rate constants of some important reactions up to some orders of magnitude. 